\begin{tabular}{|c|l|}
\hline Title & Intrinsic single and multiple pul se laser-induced damage in silicate glasses in the femtosecond-to-nanosecond region \\
\hline Author(s) & Efimov, Oleg; Juodkazis, Saulius; Misawa, Hiroaki \\
\hline Citation & $\begin{array}{l}\text { PHY SICAL REVIEW A, 69, 042903 } \\
\text { https://doi.org/L0.1103/PhysRevA.69.042903 }\end{array}$ \\
\hline Issue Date & 2004 \\
\hline Doc URL & http://hdl.handle.net/2115/5896 \\
\hline Rights & Copyright $\odot 2004$ A merican Physical Society \\
\hline Type & article \\
\hline File Information & PRA 69.pdf \\
\hline
\end{tabular}

Instructions for use 


\title{
Intrinsic single- and multiple-pulse laser-induced damage in silicate glasses in the femtosecond-to-nanosecond region
}

\author{
Oleg Efimov \\ HRL Laboratories, LLC, 3011 Malibu Canyon Road, Malibu, California 90265, USA \\ Saulius Juodkazis* \\ Core Research for Evolution Science \& Technology (CREST), Japan Science \& Technology Corporation, \\ Ecosystem Engineering Department, The University of Tokushima, 2-1 Minamijosanjima, Tokushima 770-8506, Japan \\ Hiroaki Misawa \\ Core Research for Evolution Science \& Technology (CREST), Japan Science \& Technology Corporation, \\ Research Institute for Electronic Science, Hokkaido University, CRIS Building, Sapporo 001-0021, Japan
}

(Received 14 July 2003; revised manuscript received 20 January 2004; published 21 April 2004)

\begin{abstract}
We show that the threshold power density of the intrinsic laser-induced damage in borosilicate glass at $\sim 1 \mu \mathrm{m}$ wavelength does not depend on pulse duration from $2 \times 10^{-13}$ to $3 \times 10^{-8} \mathrm{~s}$ and has the same value for both single- and multiple-pulse exposure of the sample. This indicates that the mechanism of the intrinsic damage in glasses involves a collective response of a certain volume in the dielectric as a whole, such as "dielectric-metal" phase transition, rather than a process of individual generation and accumulation of electrons, such as multiphoton, tunneling, or avalanche. Also, we demonstrate that under femtosecond exposure the threshold of the plasma formation in transparent glasses is considerably higher than the threshold of the residual change of medium parameters.
\end{abstract}

DOI: 10.1103/PhysRevA.69.042903

PACS number(s): 79.20.Ds, 81.16.-c, 32.80.Rm, 42.70.Ce

\section{INTRODUCTION}

The laser-induced damage (LID) in transparent dielectrics under short laser pulses was revealed more that 40 years ago [1]; however, the nature of this phenomenon is still under active discussion. Most of existing theories, including the advanced avalanche model [2-6], are based on the assumption of an individual ionization of ions and an accumulation of free electrons up to a certain concentration, providing a high absorption of laser radiation simultaneously with the pulse action. This is why all these models predict a rather strong dependence of the LID threshold on the pulse duration [2-4] and a decrease of the damage threshold under multiple pulse irradiation [7-9].

It was established that the LID threshold, which is usually specified in terms of the laser fluence $\left(\mathrm{J} / \mathrm{cm}^{2}\right)$ for practical needs, depended on pulse duration as $\tau^{x}(0.3<x<0.6)$ for $\tau>10$ ps [2-4], while for shorter pulses the LID threshold tended to be constant. All the diversity of these dependencies had been explained by avalanche ionization with different contributions of multiphoton and/or tunneling excitation of electrons. Actually, for accurate comparison with the theoretical conclusions, it is necessary to be sure that only the intrinsic LID threshold is measured. This threshold is specified by the interaction of optical radiation with a material matrix when there are no subthreshold distortions of a spatial-

\footnotetext{
*Corresponding author. Present address: Research Institute for Electronic Science, Hokkaido University, CRIS Building, Sapporo 001-0021, Japan. FAX: (+81 11) 706 9359. Electronic address: saulius@es.hokudai.ac.jp
}

temporal structure of radiation in the interaction volume. The absence of such distortions is a necessary condition for correct calculation of the absolute irradiance of the LID threshold. This calculation involves data about the beam spot size and the beam instant power which, in most cases, cannot be measured directly at the moment of damage. Thus, any uncontrolled variation of a radiation structure must be excluded throughout the experiment.

To adjust to the above-mentioned requirements a few conditions have to be satisfied. First, the laser radiation pulses must have no spatial-temporal fluctuation of their intensity [10]. This is especially important for the pulses of duration longer than $100 \mathrm{ps,}$, where a single-frequency laser radiation has to be used. The application of such a single-frequency laser in experiments excludes the intensity fluctuation due to interference of longitudinal modes and maintains the statistical distribution of the glass damage thresholds within $\pm 2 \%$ accuracy of measurement [11]. The shorter pulses have much broader spectra and their radiation statistics are closer to that of white light. Conservation of the proper amplitude-phase relations between the laser modes in such pulses minimizes occasional temporal fluctuations of laser intensity and makes these pulses acceptable for the damage experiments. Second, the influence of self-focusing has to be minimized as much as possible [12]. To achieve that, special optical systems with a high numerical aperture $(>1)$ are usually employed to focus the laser radiation [5,12]. To realize such a high numerical aperture (NA), matching liquids are employed because without them, the NA is limited to values smaller than 1 and leads to strong aberrations overstating the measured values of the LID thresholds.

When only the surface damage thresholds are measured, self-focusing does not take place. Therefore, to determine the 
mechanism of the LID in this case, the dependence of the LID threshold on pulse duration was measured on the front surface of some dielectrics $[3,4,6]$. Unfortunately, that data cannot be used for a judgment about the bulk damage because the bulk and the surface of the materials have strongly different physical properties and electron energy spectra. Consequently, as a rule, the bulk and surface LID thresholds have different absolute values as well as their statistical distributions $[10,13]$.

Measurements of the bulk LID in transparent dielectrics when all the above-mentioned conditions were taken into account, were carried out in only a few studies $[5,11,12,14]$. They showed that the threshold of the LID in the borosilicate glass at $1.06 \mu \mathrm{m}$ wavelength did not depend on pulse duration in the picosecond-to-nanosecond region [14]. This indicated that there were no mobile charges in the silicate glasses under subthreshold irradiance of exposure. The analogous conclusion was drawn in the investigation of the multiple pulse damage (MPD) in silicate glasses [15]. This phenomenon reveals itself as a decrease in the damage threshold under multiple-pulse irradiation. It is usually connected with generation of defects as a result of a nonlinear excitation of electrons: breakage of chemical bonds [7], multiphoton absorption [8], or two-step excitation including harmonic generation followed by multiphoton absorption [9]. This implies that the basic reason resulting in both single- and multiplepulse LID in transparent dielectrics is considered to be the same: an individual ionization of ions and an accumulation of electron defects. However, the studies of the MPD in silicate glasses under nanosecond pulses $[15,16]$ showed that the damage threshold decrease at $1.06 \mu \mathrm{m}$ was not related to a subthreshold electron excitation. Thus, both measurement of the dependence of the intrinsic LID threshold on pulse duration and study of the MPD under nanosecond pulses indicated that there were no mobile charges in silicate glasses at $1.06 \mu \mathrm{m}$ wavelength under subthreshold irradiance of exposure.

Development of femtosecond lasers has opened up unique possibilities to extend our knowledge about LID regularities. In particular, investigations of surface LID have revealed rather close thresholds for both single- [4] and multiple- [3] pulse exposure of samples and have allowed the authors to draw a conclusion about the important role of multiphoton and/or tunneling ionization in the LID of surfaces. Unfortunately, measurements of the intrinsic LID in the bulk of dielectrics under femtosecond pulses were carried out with only a few materials [5]. Thus, up to now, the absence of data about the single- and multiple-pulse damages under tightly focused radiation in the same material over the femtosecond-to-nanosecond region has prevented comprehensive estimation of the possible mechanisms of the LID.

In this paper we have measured the intrinsic LID threshold of borosilicate glass (BK7 in Schott nomenclature) under femtosecond laser pulses at a wavelength of $0.8 \mu \mathrm{m}$. It is known that the LID threshold of wide band gap silicate glasses is changed insignificantly with wavelength in the region $0.53-1.06 \mu \mathrm{m}$ [17]. This fact allowed us to compare the thresholds at 0.8 and $1.06 \mu \mathrm{m}$ for the $0.2 \mathrm{ps}-30 \mathrm{~ns}$ pulse durations. In addition, we have studied the properties of the MPD in the same glass under tightly focused femtosecond

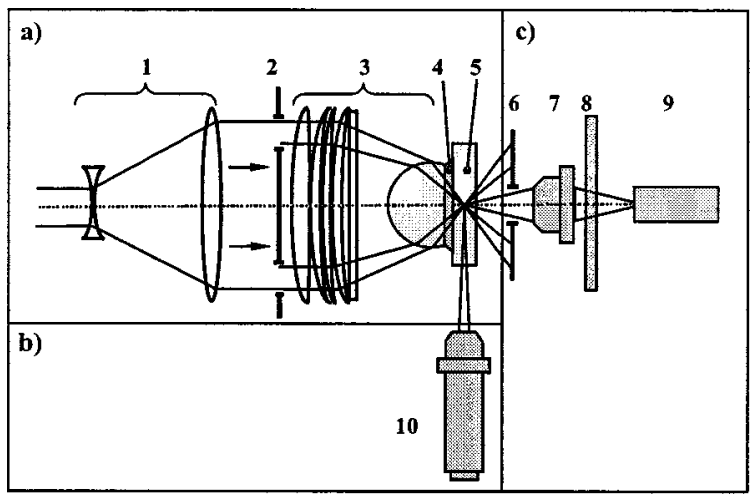

FIG. 1. Optical setup for (a) tight focusing of laser radiation into the sample, (b) plasma emission observation, and (c) dark field imaging (see details in Sec. II).

pulses and compared them with data obtained previously for nanosecond pulses [15].

\section{EXPERIMENT}

Femtosecond laser pulses were obtained from a Ti:sapphire laser which oscillated at a $\sim 1 \mathrm{kHz}$ pulse repetition rate (Tsunami with Spitfire Spectra Physics Inc.). The pulse duration was measured directly past the focal region of the optical systems using a GRENOUILLE [18] (Swamp Optics, LLC) setup and the pulse retrieval algorithm of a frequencyresolved optical gating (Femtosecond Technologies, LLC). For single-shot LID threshold measurement, the sample was moved with a speed of $\sim 0.1 \mathrm{~mm} / \mathrm{s}$ across the beam during the measurements and the pulse repetition rate was changed to $10 \mathrm{~Hz}$. This secured a single exposure of tested points which were separated from each other by a distance of about $10 \mu \mathrm{m}$; it also excluded any influence of accumulation processes on the obtained data and allowed us to evaluate the statistical distribution of the damage thresholds.

The LID threshold of the same sample of borosilicate glass BK7 [19] under femtosecond pulses was measured with two different high NA optical systems. One of them [Fig. 1(a)] was used earlier in the experiments with all pulse durations. It had an NA of 1.07. This system allowed obtaining a spot size of $0.5 \mu \mathrm{m}$ [full width at half maximum (FWHM)] for focused laser radiation at $1 \mu \mathrm{m}$ wavelength and excluding the influence of self-focusing on the results of measurements [12]. The laser beam was expanded with a telescope (1), passed a ring diaphragm (2), and was focused by the objective and glass sphere (3) into the sample (5) through an immersion oil (4). Pulse duration at the focus, measured as described above, was $(210 \pm 10)$ fs. The measurement of the LID threshold in this case was determined by visual observation of a plasma spark in the focal region in the direction perpendicular to the optical axis [Fig. 1(b)]. Because of the very small exposure area $(\sim 0.5 \mu \mathrm{m}$ in diameter) and the short time of interaction $(\sim 200 \mathrm{fs})$ the plasma emission under threshold conditions was rather low. Therefore, the observation was carried out under the microscope (10) with NA=0.4. Thus, this method gave us the plasma formation (PF) threshold. 


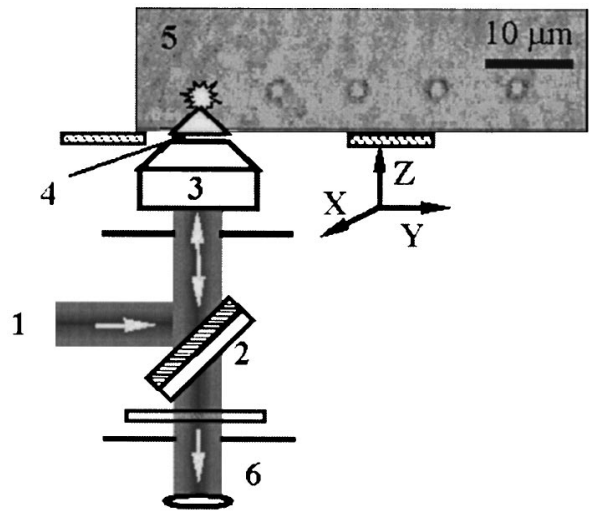

FIG. 2. Optical setup for in situ observation of the LID in the bulk of transparent dielectrics.

The other system (Fig. 2) was designed on the basis of a microscope [20] and was only used for threshold measurements with femtosecond pulses. The laser radiation (1) entered the microscope after reflection from the dichroic mirror (2) and was focused by the commercial immersion objective lens (3) into the sample (5) through an immersion oil (4). Pulse duration at the focus of this system was $(250 \pm 10) \mathrm{fs}$. The objective lens (3) had tunable NA=0.8-1.35. The spatial distribution of the intensity was measured at the focus in the bulk of the tested glass (5) and the NA of the objective was adjusted to have approximately the same spot size as in the previous optical system.

In order to measure the focal spot size at the FWHM level, the following procedure was applied. The dependence of the ablation spot diameter $D$ vs the irradiance per pulse, $I_{i n}$, was measured for a single shot exposure of BK7 glass surface. For a Gaussian pulse, the ablation threshold irradiance, $I_{t h}$, and the effective waist diameter at $1 / e^{2}$ level, $\omega$, can be determined from the dependence $I_{t h}=I_{i n} e^{-2(D / \omega)^{2}}$. We measured the ablation spot diameter with an atomic force microscope, which was additionally calibrated using a scanning electron microscope. The best fit of the atomic force microscope data to the above-mentioned equation was used to calculate the waist diameter at FWHM level, $\omega_{F W H M}$ $=(0.61 \pm 0.06) \mu \mathrm{m}$.

The threshold was defined by direct observation of the residual changes in the focal region through the dichroic mirror (2) at the exit (6) of the microscope (Fig. 2). In this optical system the same objective lens (3) with a high numerical aperture was used for both the damage to the sample and the observation of the damage. Therefore, this scheme was self-adjusted and had very high resolution and sensitivity. The sample (5) in Fig. 2 is an image of the real sample fragment with the damaged points. One can see that only diffraction spots could be seen through the microscope. This fact prevented our drawing any conclusion about the structure of the damaged areas. These images indicated only that some modification of glass parameters resulting in the observed diffraction spots occurred within a very small interaction area.

To measure the damage threshold, a line of points in the glass was irradiated with the same energy of laser pulses and

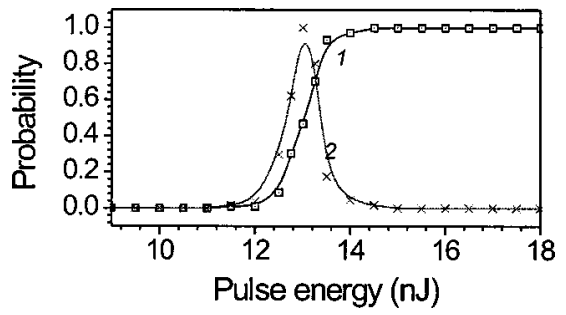

FIG. 3. Probability of the LID in the glass sample vs pulse energy measured at the entrance of the microscope (1) and its derivative (2).

recorded by a charge coupled device (CCD) camera [sample (5) in 2]. An LID probability at definite pulse energy was estimated by calculation of a relative number of the damaged points in the line. The sample was irradiated with the different pulse energies and the probability curve like that shown in Fig. 3 (curve 1) was plotted. The derivative of this curve (Fig. 3, curve 2) shows that the described method allowed reaching an accuracy of $\pm 5 \%$. The pulse energy at the focus was calibrated using a solid immersion lens [21]. Thus, this method gave us the threshold for the residual changes of the glass parameters in the focal region.

To study the properties of the MPD under tightly focused femtosecond pulses, the separate points of the sample were irradiated with multiple pulses from the above-mentioned Ti:sapphire laser oscillating at a $\sim 1 \mathrm{kHz}$ pulse repetition rate. The dark field scheme shown in Fig. 1(c) was used in the experiments. A small diaphragm (6) placed on the opposite side of the sample totally blocked the laser radiation. Therefore, only plasma emission, luminescence, or laser radiation scattered from the focal region could be observed with the CCD camera (9) through the objective with NA $=0.4$ (7). Insertion of a narrow-band $(20 \mathrm{~nm})$ reflecting filter (8) at $800 \mathrm{~nm}$ into the registration path allowed blocking any scattered radiation from the focal area while it scarcely changed the intensity of the plasma emission or the luminescence because of the very narrow bandwidth of the filter. Therefore, insertion and removal of the filter (8) was used to discriminate between the plasma radiation and the scattering from the laser pulses. The sensitivity of this dark field scheme relative to any changes in glass parameters was rather high because of using the same high intensity laser beam for both the LID and for probing the irradiated site.

\section{RESULTS AND DISCUSSION}

\section{A. Multiple pulse LID in silicate glasses}

First of all, we have studied the MPD in silicate glass BK7 under femtosecond pulses which were tightly focused in the spot size of $\sim 0.5 \mu \mathrm{m}$ [Figs. 1(a) and 1(c)]. The MPD is a cumulative process and has to be rather sensitive to the subthreshold accumulation of defects; therefore, it is very important for understanding the nature of the LID. It was found that the multiple pulse exposure of a glass sample to irradiance that was more than $65 \%$ of its single-pulse threshold of PF resulted in the appearance of a small luminous point in the focal region. This point disappeared when a 

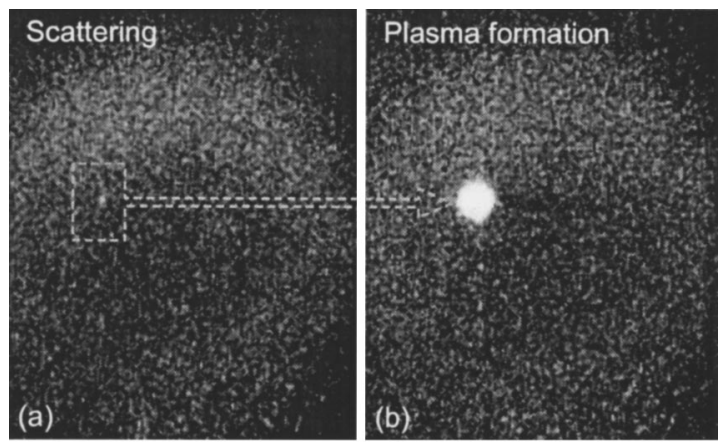

FIG. 4. (a) Dark field image of the optically modified focal spot in a glass BK7 at irradiance of $70 \%$ of the single-pulse PF threshold. This regime corresponds to the case where there is no plasma emission observed. (b) Image of plasma emission in the focal region at the PF threshold.

narrow-band filter [(8) in Fig. 1(c)] blocking only the laser radiation was inserted in the scheme. When the laser irradiance reached the PF threshold, the intensity of the plasma emission through the filter [Fig. 4(b)] was a few orders stronger than the signal of the luminous point without the filter [Fig. 4(a)]. Thus, the before-PF luminosity must have resulted from a laser radiation scattered from tiny structures produced in the focal region without PF. This meant that two different damage thresholds were observed under tightly focused femtosecond pulses. The first threshold was the irradiance resulting in the glass structure modification and the scattering from the focal region. The second threshold was the irradiance resulting in the PF in the glass volume.

Under a certain irradiance, the scattering increased in brightness with time of exposure and reached saturation without PF. This indicated that the process of inhomogeneity formation was cumulative. The level of saturation increased with the increase in laser power and finally, starting from some level, this process led to the PF. The modified structures were permanent and demonstrated the same brightness after an interruption of exposure for $30 \mathrm{~h}$. Moreover, the scattering had a linear character and the modified structures could be identified under irradiation with an intensity which was a few orders less than the intensity needed for their formation.

The appearance of such structures cannot be explained by linear heating of the glass under the multiple-pulse exposure because of the relatively low average power density of the radiation $\left(\sim 2.5 \mathrm{~kW} / \mathrm{cm}^{2}\right)$ and the high transparency of the glass. Therefore, their formation could only be connected with a nonlinear process. Specifically, such a process could be the nonlinear excitation and accumulation of electrons. However, earlier, we had studied a similar MPD in the glasses under nanosecond pulse exposure [15,16,22,23]. In that case, using different techniques, we demonstrated the absence of multiphoton absorption and electron excitation in the volumes of silicate glasses like BK7 under IR radiation, even for long nanosecond pulses. Nevertheless, under femtosecond pulses a different mechanism might be responsible for the electron generation [24]. The later research showed that in this case the ionization of the glass resulted from a two-step nonlinear process: first, a spectral broadening of the transmitting laser radiation and second, linear or two-photon absorption of the short wavelength part of this broadened supercontinuum. It was observed that the distance, which the beam traveled in the medium to attain such broadening was inversely proportional to the irradiance. This enabled us to estimate the physical possibility for electron generation under our conditions. We concluded from the above-mentioned dependence that the irradiance $I$ (units of $\mathrm{TW} / \mathrm{cm}^{2}$ ) that would be needed for such broadening after a beam had passed distance $L$ (units of $\mathrm{mm}$ ) in the glass BK7 was $I$ $\sim 4 / L$. The length of interaction in our experiments was $\sim 1 \mu \mathrm{m}$; therefore the irradiance providing for electron excitation would have to be $\sim 4 \times 10^{3} \mathrm{TW} / \mathrm{cm}^{2}$, that is about three orders of magnitude more than the observed single pulse PF threshold. This showed that as in the process for nanosecond pulses [15], the MPD under femtosecond pulses must occur without an accumulation of electron defects.

Earlier [15], we assumed that the MPD under nanosecond pulses resulted from a glass structure densification in the center of the focal region under large electrostrictional pressures. This process occurred without accumulation of electron defects and disappeared as long as self-focusing was avoided. In experiments, it was demonstrated that the MPD was not detectable by measurements with an error less than $1 \%$ under conditions of tightly focused laser pulses. Such conditions were used to determine the single-pulse damage thresholds in the same glass [11,14]. Thus, the intrinsic single- and multiple-pulse damage thresholds of glass had the same value within $1 \%$ accuracy under nanosecond pulses.

A striking similarity was revealed in the processes of MPD in both nanosecond and femtosecond regions of pulse duration at $\sim 1 \mu \mathrm{m}$ wavelength. Small areas with inhomogeneities were found. The signals from them were saturated, and then, starting from a certain intensity, that process led to PF. The inhomogeneities were very stable over time and could be found even a few days after exposure. These data suggested that the same process - densification-could be responsible for the material modification before the $\mathrm{PF}$ in both regions. However, an electrostrictional pressure resulting in densification under nanosecond pulses cannot arise under femtosecond pulses because of the very short time of interaction. Therefore, some other process must be responsible for both the structure modification and the PF under elevated intensities. To discuss the possible mechanisms of this process, the obtained data about the MPD are not enough; they must be combined with results about singlepulse damage in the same material, and this will be considered in the following section.

To summarize, in this section we have shown the existence of two LID thresholds for tightly focused femtosecond pulses: the first was the multiple-pulse threshold of the glass modification without PF, and the second, which was about 1.5 times higher, was the single-pulse threshold of PF. This means that the optically recognizable modification of the glass can be recorded with femtosecond pulses without total ionization of the focal region. To the best of our knowledge, this is the first observation of damage without PF under short laser pulses and this process can be used in various applications. 


\section{B. Dependence of intrinsic LID threshold on pulse duration}

It was mentioned in the preceding section that two optical systems were used to measure the intrinsic LID threshold of the sample of borosilicate glass BK7. First, the PF threshold was measured with the optical system shown in Figs. 1(a) and 1(b), which gave us the value of $9.8 \mathrm{TW} / \mathrm{cm}^{2}$. It is important that the same scheme [Fig. 1(a)] was used to focus laser radiation for both the measurement of the PF threshold and the study of the MPD. It allowed accurate comparison of the results in both cases and permitted calculation of the threshold of the glass modification without PF under multiple-pulse exposure. This threshold was $\sim 6.4 \mathrm{TW} / \mathrm{cm}^{2}$. Because of the considerably lower threshold of the glass modification, we repeated the measurements of the LID with the other scheme (Fig. 2) which was rather sensitive to the residual changes in the glass. These measurements gave us the single-pulse threshold of the residual changes of glass parameters in the focal region, namely, $6.6 \mathrm{TW} / \mathrm{cm}^{2}$. Thus, like the results in the nanosecond region [15], the single- and multiple-pulse damage thresholds of glass under tightly focused femtosecond pulses have the same value within the accuracy of the measurements. An even more important result is that this value was found equal to the intrinsic LID threshold of the glass under long laser pulses [11,14].

Indeed, let us compare the complete set of data in the femtosecond-to-nanosecond region. The systematic measurements of intrinsic LID in silicate glass BK7 were begun in 1996. Investigation showed [11] that the intrinsic LID thresholds of this glass at $1.06 \mu \mathrm{m}$ wavelength did not depend on laser irradiance from $4 \times 10^{-10}$ to $3 \times 10^{-8} \mathrm{~s}$ and equaled $6.5 \mathrm{TW} / \mathrm{cm}^{2}$. A special system with an optical deflector was used to cut pulses of $0.38-32$ ns out of single-frequency Nd:glass laser pulses. The optical system described in the preceding section [Fig. 1(a)] was used in the experiments. Later, the LID threshold of the same sample of borosilicate glass was measured for picosecond laser pulses (4 $\times 10^{-11} \mathrm{~s}$ ) focused with the same optical system [14]. The pulses were delivered by a mode-locked Nd:YAG (where YAG stands for yttrium aluminium garnet) laser with stabilized emission spectrum and high reproducibility of spatial and temporal parameters of radiation. As a result, practically the same value of the LID threshold, namely $5.8 \mathrm{TW} / \mathrm{cm}^{2}$, was observed for the picosecond duration pulses. Finally, the threshold of the same glass under femtosecond pulses was obtained in the present work.

Figure 5 shows a summary of the data for a single-pulse LID in the bulk of the same sample of the BK7 glass measured with different pulse durations under tight focusing conditions. One can see that the LID threshold value was found to be constant with an average value of $\sim 6.4 \mathrm{TW} / \mathrm{cm}^{2}$ over the five orders of magnitude of the laser pulse durations. This result shows that the intrinsic LID is not connected to the generation rate of electrons, their accumulation, and the ensuing ionization of the focal volume. The independence of the LID threshold on pulse duration rather implies that the photomodification of the dielectric in the focal region occurs at the threshold irradiance in a "switch-on" manner, i.e., without accumulation. The same conclusion results from the equality of the intrinsic single- and multiple-pulse thresholds
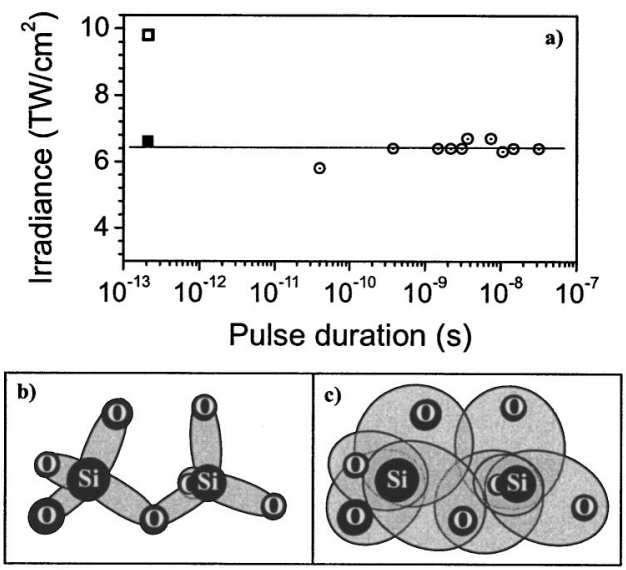

FIG. 5. (a) Threshold of the single-pulse LID in borosilicate glass vs pulse duration. For the 200 fs pulse, the $\square$ marker depicts the PF threshold, while the $\square$ marker depicts the residual modification threshold. The $\odot$ markers depict the previously obtained data $[11,14]$. (b) and (c) Schematic representations of two $\mathrm{SiO}_{4}$ tetrahedrons under a low-intensity light field and at the condition of dielectric-metal phase transition, respectively.

for both femtosecond and nanosecond tightly focused pulses. Earlier we put forward a hypothesis [12] that transition from the nonabsorbing to the highly-absorbing state proceeds according to the Mott dielectric-metal transition [25] scenario at the threshold laser irradiance. In accordance with this hypothesis, at a certain irradiance, the orbits of the valence electrons inside a dielectric [Fig. 5(b)] swell out and overlap [Fig. 5(c)] in the high-intensity electric field of a light wave with field strength of $\sim 5 \times 10^{7} \mathrm{~V} / \mathrm{cm}$. Under this scenario that transition, in fact, brings about a metalliclike state in the dielectric, identical to a dielectric-metal phase transition.

Thus, in this section we have proved the existence of the two damage thresholds which were found in the preceding section, even under single-pulse exposure of the glass. The other important result was that the threshold power densities of the intrinsic single- and multiple-pulse LID in glass were found equal to each other (i.e., constant) and did not depend on pulse duration over the range from $2 \times 10^{-13}$ to 3 $\times 10^{-8} \mathrm{~s}$.

\section{Qualitative model of LID in silicate glasses}

Taking into account all the data including the single- and multiple-pulse damage over the femtosecond-to-nanosecond laser pulses, we propose the following picture of the LID of transparent dielectrics. Electrostrictional self-focusing occurs under nanosecond laser pulses which were focused in spots of large diameter [23]. This results in the collapsing of the laser beam into a point [Fig. 6(a)]. The increase of intensity up to the phase-transition threshold value in a collapse point switches the medium to the "metal-like" state with resultant heating of this location. It is well known that self-focusing results in the motion of a collapse point along an optical axis with high speed under bell-shaped laser pulses [26]. Therefore, each point of the resulting track subjected to the phase transformation is irradiated only during a small fraction of 


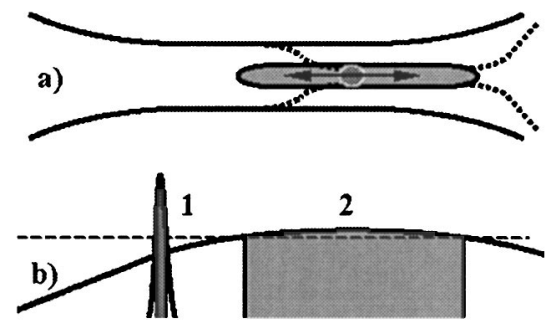

FIG. 6. Schematic representations of processes resulting in single- and multiple-pulse LID in silicate glasses. (a) A pulse waist. (b) Temporal envelopes of femtosecond (1) and picosecond or nanosecond (2) pulse. Dashed line is the threshold of dielectricmetal phase transition.

the pulse duration and the absorbed energy is not enough for the PF. However, this energy can be enough to cause formation of a small structural inhomogeneity via heating/melting and fast cooling/quenching of that waveguidelike track. This process leads to an increase in the optical inhomogeneity in the focal region under multiple-pulse exposure and to a decrease of the self-focusing threshold. In the experiments, these inhomogeneities became apparent as a growing schlieren signal under multiple-pulse exposure [15].

At higher intensity, heating causes ionization of the entire track. This is why the damage region under threshold conditions has the shape of a long filament with diameter $<1 \mu \mathrm{m}$ even for the focused beam diameters of $30 \mu \mathrm{m}$ [12]. The focusing of the nanosecond pulses into the spots of smaller sizes weakens the self-focusing influence and reduces the length of the track with a corresponding decrease in the effect of MPD [15]. Finally, this effect disappears completely under tight focusing conditions because the point of irradiation does not move. In this case, for picosecond-tonanosecond pulses [Fig. 6(b), pulse 2], even a small exceeding of the phase transition threshold results in large absorbed energy after "metallization" of the dielectric and yields to a rapid PF from the first pulse. Therefore, the thresholds of the single- and multiple-pulse LIDs under tightly focusing conditions become equal to each other and the effect of MPD disappears completely.

Because of the very low energy of tightly focused femtosecond pulses needed for the LID in the glass $(\sim 4.8 \mathrm{~nJ})$, the threshold energy absorbed during the phase transition [Fig. 6(b), pulse 1] is not enough to heat, melt, evaporate, and transfer the medium to the plasma state. Therefore, a process of glass structure modification occurs similar to the process under nanosecond pulses focused in the spots of large diameter. This results in a chance to observe the glass parameter modification over some interval of intensity before the PF [Fig. 4(a)], even for tightly focused pulses. Nevertheless, the thresholds of the first observable single- and multiple-pulse damage in glass under tightly focused pulses have the same values regardless of the pulse duration.

The process described in this section proposes that the glass structure modification occurs due to heating/melting and quenching of a small volume in the glass. This explains why the schlieren signals observed in the study of the MPD in silicate glasses under nanosecond pulses at $1.06 \mu \mathrm{m}$ [15], had a thermal nature. Further, this process implies that glass subjected to such heating and quenching will have lower refractive index, which, in turn, will result in an appearance of long, thin, transparent filaments under nanosecond pulses focused in the spots of large diameter, and in a scattering from the modified structures under tightly focused femtosecond pulses. Thus, in accordance with this model, the modified structures observed under multiple-pulse exposure before the PF should represent the areas with refractive indices lower than the refractive index of the surrounding glass.

\section{CONCLUSIONS}

In conclusion, we have demonstrated that the intrinsic single- and multiple-pulse LID thresholds of industrial borosilicate glass BK7 specified in terms of the laser irradiance (units of $\mathrm{W} / \mathrm{cm}^{2}$ ) are equal to each other and do not depend on pulse duration over the range from $2 \times 10^{-13}$ to 3 $\times 10^{-8} \mathrm{~s}$. This indicates that the mechanism of the intrinsic damage of glasses involves a collective response of a certain volume in the dielectric as a whole, like "dielectric-metal" phase transition, rather than a process of individual generation and accumulation of electrons, such as multiphoton, tunneling, or avalanche. Local phase transition in the glass followed by a fast heating and cooling results in modification of the material without plasma formation and in a decrease in the plasma formation threshold under multiple femtosecond and nanosecond pulse exposures.

\section{ACKNOWLEDGMENTS}

This work was partially supported by the Satellite Venture Business Laboratory of the University of Tokushima, which provided a visit grant to O.E. Also, S.J. acknowledges support by U.S.A. Aerospace R\&D Contract No. F62562-03-P0208 AOARD 02-35.
[1] P. D. Maker, R. W. Terhune, and C. M. Savage, in Proceedings of the Third International Congress, Paris 1963, edited by P. Grivet and N. Bloembergen (Columbia University Press, New York, 1964), p. 1559.

[2] N. Bloembergen, IEEE J. Quantum Electron. QE-10, 375 (1974)

[3] B. C. Stuart, M. D. Feit, S. Herman, A. M. Rubenchik, B. W.
Shore, and M. D. Perry, Phys. Rev. B 53, 1749 (1996).

[4] A. C. Tien, S. Backus, H. Kapteyn, M. Murnane, and G. Mourou, Phys. Rev. Lett. 82, 3883 (1999).

[5] C. B. Schaffer, A. Brodeur, and E. Mazur, Meas. Sci. Technol. 12, 1784 (2001).

[6] T. Q. Jia, Z. Z. Xu, X. X. Li, R. X. Li, B. Shuai, and F. L. Zhao, Appl. Phys. Lett. 82, 4382 (2003). 
[7] A. E. Chmel, Mater. Sci. Eng., B B49, 175 (1997).

[8] S. Jones, P. Braunlich, R. Casper, X. Shen, and P. Kelly, Opt. Eng. 28, 1039 (1989).

[9] S. K. Balitskas, P. I. Barkyavichyus, I. A. Gul'binas, A. A. Zhilenis, I. P. Lukoshyus, E. K. Maldutis, S. V. Sakalauskas, and S. I. Yatsinavichyus, Izv. Akad. Nauk SSSR, Ser. Fiz. 54, 1552 (1990).

[10] L. B. Glebov, O. M. Efimov, G. T. Petrovskii, and P. N. Rogovtsev, Sov. J. Quantum Electron. 14, 226 (1984).

[11] O. M. Efimov, V. S. Popikov, and M. J. Soileau, J. Opt. Technol. 63, 120 (1996)

[12] L. B. Glebov and O. M. Efimov, Bull. Acad. Sci. USSR, Phys. Ser. (Engl. Transl.) 49, 94 (1985).

[13] L. B. Glebov, O. M. Efimov, N. V. Nikonorov, and G. T. Petrovskii, Sov. J. Quantum Electron. 15, 1410 (1985).

[14] O. M. Efimov, L. B. Glebov, V. S. Popikov, and M. J. Soileau, in Proceedings of the SPIE, edited by A. A. Andreev and V. M. Gordienko (SPIE, Bellingham, WA, 1996), Vol. 2770, pp. 162167.

[15] O. N. Bosyi and O. M. Efimov, Quantum Electron. 26, 718 (1996).

[16] O. N. Bosyi and O. M. Efimov, Quantum Electron. 26, 710 (1996).

[17] L. B. Glebov, O. M. Efimov, G. T. Petrovskii, and P. N.
Rogovtsev, Sov. J. Quantum Electron. 13, 972 (1983).

[18] P. ÓShea, M. Kimmel, X. Gu, and R. Trebino, Opt. Lett. 26, 932 (2001).

[19] Actually, the same sample was used to absolutely guarantee the reliability of the measurements. We found that when samples of the same glass were taken from a different melting, the values of the LID thresholds differed by no more than $10 \%$.

[20] M. Watanabe, S. Juodkazis, H. Sun, S. Matsuo, and H. Misawa, Appl. Phys. Lett. 77, 13 (2000).

[21] S. Matsuo and H. Misawa, Rev. Sci. Instrum. 73, 2011 (2002).

[22] L. B. Glebov, O. M. Efimov, and G. T. Petrovskii, Sov. J. Quantum Electron. 16, 1245 (1986).

[23] O. N. Bosyi, O. M. Efimov, and A. M. Mekryukov, in Proceedings of the SPIE, edited by A. M. Bonch-Bruevich, V. I. Konov, and M. N. Libenson (SPIE, Bellingham, WA, 1991), Vol. 1440, pp. 57-62.

[24] O. M. Efimov, S. V. Garnov, L. B. Glebov, K. Gabel, S. Grantham, M. Richardson, and M. J. Soileau, J. Opt. Soc. Am. B 15, 193 (1998).

[25] K. W. Boer, Survey of Semiconductor Physics (Van Nostrand Reinhold, New York, 1990), Vol. I.

[26] M. Loy and Y. Shen, IEEE J. Quantum Electron. QE-9, 409 (1973). 